\title{
Entanglement Between Qubits Interacting with Thermal Field
}

\author{
E.K. Bashkirov a and M.S. Mastyugin \\ Samara State University, 1 Academician Pavlov Str. Samara 443011, Russia
}

\begin{abstract}
We have investigated the entanglement between two dipole coupled two-level artificial atoms (superconducting qubits, ion, spins etc.). The model, in which only one atom is trapped in an lossless cavity and interacts with single-mode thermal field, and the other one can be spatially moved freely outside the cavity has been carried out. We have considered the effect of the atomic coherence on the entanglement behavior. We have shown that a thermal field might cause high entanglement between the atoms both for coherent and incoherent initial atomic states only for small values of cavity mean photon number. We have also derived that the degree of entanglement is weakly dependent on the strength of dipole-dipole interaction for coherent initial states. In the considered model the atoms would get entangled even when both atoms are initially in the excited state.
\end{abstract}

Keywords: entanglement, cavity QED, negativity, thermal field, atomic coherence

Entanglement between separate quantum systems is one of the key problem in quantum mechanics. The interaction between the environment and quantum systems can lead to decoherence, But it may also be associated with the formation of non-classical effects such as entanglement. Kim et al. showed [1] that a chaotic field with minimal information can entangled two two-level atoms interacting with one-mode thermal field in a lossless cavity. The practical applications in quantum information processing require engineering entangled atoms so this expects operable atoms which can be moved to distance without losing of information. We have considered the scheme in which is only one atom is trapped in a cavity, and the other one can be spatially moved freely outside the cavity.

We consider two two-level artificial atoms (superconducting circuits, neutral atoms and ions, spins or hibrid qubits [2]) and one-mode quantum thermal cavity field. The first atom is trapped in a lossless microcavity and resonantly interacts with the cavity field of the frequency $\omega$. The second atom lies beside the first atom out of the cavity. We assume that the distance between atoms can compare with a wavelength of atomic transition. In this case the dipole-dipole interaction should be included. The Hamiltonian of this system can be written as

$$
H=(1 / 2) \mathrm{h} \omega\left(\sigma_{1}^{z}+\sigma_{2}^{z}\right)+\mathrm{h} \omega a^{+} a+\mathrm{h} g\left(\sigma_{1}^{+} a+a^{+} \sigma_{1}^{-}\right)+\mathrm{h} J\left(\sigma_{1}^{+} \sigma_{2}^{-}+\sigma_{1}^{-} \sigma_{2}^{+}\right),
$$

where $(1 / 2) \sigma_{i}^{z}$ is the inversion operator for the $i$ th atom $(i=1,2), \sigma_{i}^{+}=|+\rangle_{i i}\langle-|$, and $\sigma_{i}^{-}=|-\rangle_{i i}\langle+|$ are the transition operators between the excited $|+\rangle_{i}$ and the ground $|-\rangle_{i}$ states in the $i$ th atom, $a^{+}$and $a$ are the creation and the annihilation operators of photons of the cavity mode, $g$

\footnotetext{
${ }^{a}$ Corresponding author: bash@samsu.ru
} 
is the coupling constant between atom and the cavity field and $J$ is the coupling constant of the dipole interaction between the atoms. We consider that the initial state of each atoms is a coherent superposition of the two levels, that is,

$$
\left|\Psi_{1}(0)\right\rangle=\cos \theta_{1}|+\rangle+e^{i \varphi_{1}} \sin \theta_{1}|-\rangle,\left|\Psi_{2}(0)\right\rangle=\cos \theta_{2}|+\rangle+e^{i \varphi_{2}} \sin \theta_{2}|-\rangle .
$$

Here $\theta_{1}$ and $\theta_{2}$ denote the amplitudes of the polarized atoms, and $\varphi_{1}$ and $\varphi_{2}$ are relative phases of two atoms, respectively. The initial cavity mode state are assumed to be the thermal one-mode state

$$
\rho_{F}(0)=\sum_{n} p_{n}|n\rangle\langle n| .
$$

The weight functions are $p_{n}=\bar{n}^{n} /(1+\bar{n})^{n+1}$, where $\bar{n}_{i}$ is the mean photon number in the $i$ th cavity mode.

We have obtained the exact solution for model under consideration. Calculating the time behaviour of negativity we have derived, firstly, that entanglement can be induced by thermal field for all initial atomic states. The more significant result is that the atomic entanglement may be induced by thermal field when both the atoms are prepared in their excited states. Secondly, the maximum degree of entanglement is slightly enhanced owing to the atomic coherence. With increasing of the mean photon number the value of atom-atom negativity decreases. But for coherent states this decreasing is much sharper. For large mean photon number the entanglement does not occur.

\section{References}

1. M.S. Kim, J. Lee, D. Ahn, P.L. Knight, Phys. Rev. A 65., No 4. P. 040101 (2002)

2. I. Buluta, S. Ashhab, F. Nori, Rep. Prog. Phys. 74, No 10. P. 104401 (2011) 